\title{
ROLE OF ANALOGY METHOD IN FORMING THE COMPETENCE OF FUTURE TEACHERS
}

\section{O.K. Kuvandikov}

Samarkand State University, Uzbekistan

\section{M.K. Salakhitdinova}

Samarkand State University, Uzbekistan

\section{M.A.Eshmirzaeva}

Karshi Civil Engineering Institute, Uzbekistan

\section{ABSTRACT}

In this work, for the first time, the possibility of using the analogy method in solving scientific and pedagogical problems in the light of personnel training is analyzed. It is shown that the use of analogy is useful for quick assimilation of educational material and in solving physical problems. In particular, the possibility of using the concepts and methods of geometric optics in the design of electrical devices by analogy with the corresponding optical instruments is shown.

KEYWORDS: - Formation of competencies, government program, radical reform of education, renewal of knowledge, dynamic analogies, mechanics, optics.

\section{INTRODUCTION}

The government program adopted in our country substantiates the need for its radical reform, shows the strategic directions of development and the main expected results.

The new paradigm of education should be focused on the formation of needs for constant replenishment and renewal of knowledge, improvement of skills and abilities, their consolidation and transformation into competencies. In particular, the knowledge paradigm of education should be revised from the standpoint of competent approach $[1,2]$.

The formation of competencies is one of the areas of modernization of general education. The modern student of higher education must not only master certain knowledge, skills and abilities, he must be able to accept the knowledge gained in his activities, in some non-standard situations.

The competence of student presupposes the manifestation of whole spectrum of his personal qualities in relation to the competence. "The 
CURRENT RESEARCH JOURNAL OF PEDAGOGICS 2(12): 30-35, December

2021 DOI: https://doi.org/10.37547/pedagogics-crjp-02-12-07

ISSN 2767-3278

(C)2021 Master Journals

Crossref do: 81 Google

Accepted 10 $10^{\text {th }}$ December, 2021 \& Published 15 th December, 2021

concept of competence includes not only cognitive and operational-technological components, but also motivational, ethical, social and behavioral", i.e. competence is always colored by the qualities of particular student. These qualities can be whole fan - from semantic and associated with goal-setting (why do I need this competence), to reflexive-evaluative (how successfully I apply this competence in life).

Studies on analogies are of great importance in the formation of the competencies of future physicists because they lead to the mutual enrichment of different disciplines, and also allow us to see the general physical laws that underlie seemingly different phenomena $[3,4]$.

Historically, one of the origins of quantum mechanics was the parallels established by Hamilton between geometric optics and mechanics. These forgotten analogies were attracted by de Broglie [5] into modern physics and with their help the first steps of quantum (wave) mechanics were made. E. Schrödinger [6] in the construction of quantum mechanics was based on the optical-mechanical analogy of Hamilton.
As methodological device and tool for scientific research, analogy was the subject of serious interest on the part of Maxwell [7, 8]. J. Maxwell compared the classical theory of electromagnetism created by him with the hydrodynamics of incompressible fluids and emphasized the importance of this approach in science: "To form physical concepts, get used to the existence of physical analogies. By physical analogy, I mean that particular similarity between the laws of any two fields of science, thanks to which one of them is an illustration for the other. " In the future, it was precisely the analogy that was destined to play an outstanding role in Maxwell's research on the theory of electromagnetism. The analogies that exist between electrical, mechanical, acoustic and other oscillatory systems have long been successfully used by physicists and technicians.

K.Maxwell [7] developed the theory of the electromagnetic field using analogy as method of scientific research. The following Table 1 shows the analogy between the quantities of mechanics and electrodynamics.

Table 1. Analogy between the quantities of mechanics and electrodynamics

\begin{tabular}{|l|l|}
\hline Mechanics & Electromagnetism \\
\hline Stream & Current power \\
\hline The linear velocity of the vortex & Magnetic field strength \\
\hline The average speed of the vortex & Magnetic susceptibility \\
\hline $\begin{array}{l}\text { The kinetic energy of vortex 's } \\
\text { rotational motion }\end{array}$ & Magnetic field energy \\
\hline Elasticity constant & Dielectric singularity \\
\hline
\end{tabular}

On this basis, he derived the following equations:

$$
\nabla \stackrel{\mathrm{I}}{E}=4 \pi \rho, \nabla \stackrel{\mathbf{B}}{\mathrm{B}}=0
$$




$$
\text { or } \quad \nabla \stackrel{\mathrm{r}}{E}=-\frac{1}{c} \frac{\partial \stackrel{\mathrm{L}}{\partial}}{\partial t}, \quad \nabla \stackrel{\mathrm{r}}{B}=\frac{1}{c} \frac{\partial \stackrel{\mathrm{L}}{\partial t}}{\partial t}+\frac{4 \pi}{c} \stackrel{\mathrm{r}}{j}
$$

here $\nabla$-nabla operator, $\stackrel{1}{E}, \stackrel{1}{B}$ - electric and magnetic field voltage vector, $c$ - speed of light in vacuum, $\stackrel{1}{j}$ - current density.

Based on these equations, he created the electromagnetic theory of light.

Continuing K. Maxwell's theory, in 1883, the English physicist O. Xevisaydo solved the properties of electric and magnetic fields in new way, using the method of analogy to the electromagnetic field, introducing the concept of magnetic charge. Its equations are as follows:

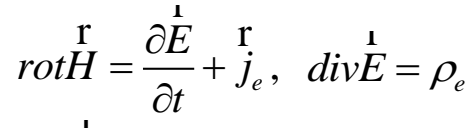

$$
\begin{aligned}
& \operatorname{rot} \stackrel{\mathrm{r}}{E}=-\frac{\partial \stackrel{\mathrm{H}}{\partial}}{\partial t}-\stackrel{\mathrm{r}}{j_{m}}, \quad \operatorname{div} \stackrel{\perp}{H}=\rho_{m}
\end{aligned}
$$

here $\dot{j}_{e}$ - electric current density, $\dot{j}_{m}$ - magnetic current density, $\rho_{e}$-electric charge, $\rho_{m}$-magnetic charge density.

R. Feynman [6] showed that it is possible to understand physical phenomena of different nature through electrostatic analogy:

1. That the electrostatic matter is the same as in the heat statistics when the heat flux is constant;

2. Tensile strength or membrane potential, uniformity of Poisson and Laplace equations;

3. That the diffusion of neutrons is also solved by the Poisson and Laplace equations;

4. Laminar flow of fluid;

5. Illumination;

6. The passage of electrons through semiconductors.

It is difficult to get all the information about the surrounding world, so it is necessary to get general information about the physical world. There are 3 reasons for this: first, there are general physical principles that can be applied to any event. For example, mass, energy, momentum, momentum, and so on. Second, many complex phenomena, such as the compression of solids, are mainly determined by electromagnetic and quantum mechanical forces. Third, there is remarkable compatibility analogy, whereas equations in different physical conditions have the same appearance, only the characters (nouns) used are different, the mathematical equation is the same. For example, the vibration of disc, the motion of body attached to spring, the tensile force of string, the rise of the liquid level in capillary vessel, the vibration contour of open tube, the hydrometer, the capacitance and the inductance. It can be considered as the simplest harmonic oscillator of these systems. They are, at first glance, subject to the laws of harmonic motion to deviate from their equilibrium or quiescent state, and the equation of harmonic motion can be applied to all [12].

Methods based on the use of dynamic analogies [9] make it possible to simplify calculations and make both intermediate studies and more visible. their results. The power of these methods is revealed mainly in the analysis and calculations of complex systems in which, for example, mechanical, electrical and acoustic vibrations occur simultaneously. Analogies are 
CURRENT RESEARCH JOURNAL OF PEDAGOGICS 2(12): 30-35, December

2021 DOI: https://doi.org/10.37547/pedagogics-crjp-02-12-07

ISSN 2767-3278

(C)2021 Master Journals

Crossref do: 81 Google

Accepted 10 $10^{\text {th }}$ December, 2021 \& Published $15^{\text {th }}$ December, 2021

useful in analysis in unexplored areas. By analogy can be compared to unknown system with previously studied system.

The most widespread oscillatory system is the electrical circuit, although this is often overlooked. Knowledge in the field of electrical circuits, with the help of analogies, can be applied to solving problems in the field of mechanics and acoustics. This replaces the mechanical or acoustic system with similar electrical system. There is deep and non-trivial analogy between the basic concepts of mechanics and optics [10], which makes it possible to compile the following "dictionary" (see Table 2) for translating the statements of mechanics into the language of optics and vice versa.

The analysis of the motion of charged particles in electrostatic fields does not present serious mathematical difficulties only for fields of the simplest configurations (flat capacitor, field of point charge, field of charged cylindrical conductor).

Table 2. Analogy between mechanics and optics.

\begin{tabular}{|c|c|}
\hline Mechaics & Optics \\
\hline Material point & Wave packet \\
\hline Trajectory & beam \\
\hline Speed, $\vartheta$ & Group speed, $\vartheta$ \\
\hline $\begin{array}{c}\text { There is no simple analogy } \\
\text { Potential energy U }=\mathrm{u}(\mathrm{x}) \text { as } \\
\text { coordinate functions }\end{array}$ & $\begin{array}{c}\text { Refractive index of the coordinate function (or } \\
\text { phase velocity) }\end{array}$ \\
\hline Energy E & Frequency, or $E=E(v)$ \\
\hline $\begin{array}{c}\text { Trajectory from the Maupertuis principle } \\
\sqrt{E-U d s}=\min (1)\end{array}$ & $\begin{array}{c}\text { Beam from the Fermat principle } \int \frac{d S}{V}=\min \\
(2)\end{array}$ \\
\hline
\end{tabular}

However, in all practically interesting cases, the electric field turns out to be so complex that even the expression for the potential cannot be represented in finite form using elementary functions. As result, the integration of the equations of motion becomes extremely complicated and can only be carried out numerically.

There is general approach to such problems, which for wide class of systems allows you to determine the shape of the trajectory. The method is based on far-reaching analogy between the motion of charged particle and the propagation of light rays in transparent refractive medium [11]. As is known, in the framework of geometrical optics, the propagation of light beam in any inhomogeneous medium can be described on the basis of the law of refraction of light. To do this, you need to know the values of the refractive index along the entire path of the light beam. Therefore, in optics it is conventionally assumed that the refractive index of vacuum is equal to unity. It is easiest to verify the existence of the above analogy by analyzing one simple example.

Suppose that charged particle moves in space, in which there is potential jump at some boundary (Fig. 1, 2). Such potential jump, of course, cannot be performed technically, since it corresponds to 
CURRENT RESEARCH JOURNAL OF PEDAGOGICS 2(12): 30-35, December

2021 DOI: https://doi.org/10.37547/pedagogics-crjp-02-12-07

ISSN 2767-3278

(C)2021 Master Journals

Crossref do: 81 Google

Accepted 10 ${ }^{\text {th }}$ December, $2021 \&$ Published 15 th December, 2021

an infinitely large value of the field strength. The best approximation would be system consisting of two closely spaced sheets of extremely thin metal foil, transparent to the particles under consideration and charged to the corresponding potentials. Passing through the interface, charged particle experiences the action of force directed along the normal to this interface. Therefore, the normal component of the velocity changes, while the tangential component remains unchanged. The last condition gives

$$
v_{1} \sin \alpha=v_{2} \sin \beta
$$

where $v_{1}$ and $v_{2}$ the values of the particle velocity before and after passing through the interface, and the angles $\alpha$ and $\beta$, by analogy with optics, can be

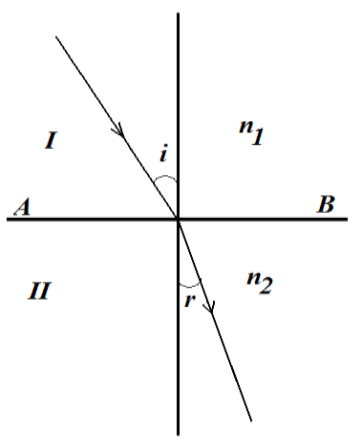

Fig.1. Refraction of beam of light at the border of two media with different refractive indices.

The existence of the considered analogy makes it possible to use the concept of methods of conventional geometric optics in new field and to design electrical devices by analogy with the corresponding optical instruments. The most striking example of the fruitfulness of this approach was the creation of electron microscope, device that allows one to obtain images of objects in electron beams with called the angle of incidence and the angle of refraction.

$$
\frac{\sin \alpha}{\sin \beta}=\frac{\vartheta_{2}}{v_{1}}
$$

If, as usual, we assume that the value of the particle's velocity is determined by the value of the potential at given point, then equality (4) can be written in the following form:

$$
\frac{\sin \alpha}{\sin \beta}=\sqrt{\frac{U_{1}}{U_{2}}}
$$

the written equality completely coincides with the usual formulation of the law of refraction in optics.

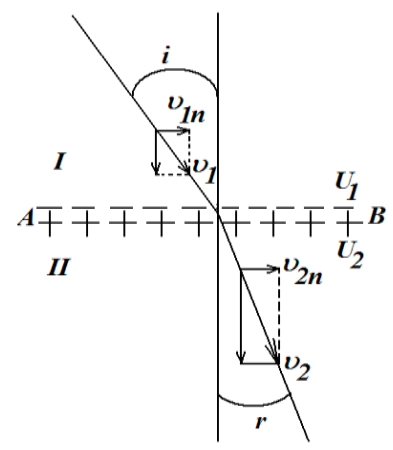

Fig 2. Application of an electron beam at the interface of two media with different potentials.

resolving power several orders of magnitude higher than the resolving power of conventional microscope.

In general terms, the design of an electron microscope, designed to obtain images of such objects in transmitted electron beams, repeats the design of an optical microscope. 
CURRENT RESEARCH JOURNAL OF PEDAGOGICS 2(12): 30-35, December

2021 DOI: https://doi.org/10.37547/pedagogics-crjp-02-12-07

ISSN 2767-3278

(C)2021 Master Journals

Crossref do: 81 Google

Accepted 10 $10^{\text {th }}$ December, 2021 \& Published $15^{\text {th }}$ December, 2021

\section{Conclusion}

So, in this work, for the first time, the possibility of using the analogy method in solving scientific and pedagogical problems in the light of personnel training is analyzed. It is shown that the use of analogy is useful for quick assimilation of educational material and in solving physical problems. In particular, the possibility of using the concepts and methods of geometric optics in the design of electrical devices by analogy with the corresponding optical instruments is shown; the analogy between the processes of transfer of particles, thermal conductivity and diffusion and the transfer of electric charges, etc. is considered.

\section{REFERENCES}

1. Resolution of the President of the Republic of Uzbekistan dated March 19, 2021 "On measures to improve the quality of education and development of scientific research in the field of physics." Tashkent, 2021; Resolution of the President of the Republic of Uzbekistan "On measures to further develop the system of higher education" No. PP-2909, April 20, 2017.

2. O.K.Kuvandikov National program of the Republic of Uzbekistan for personnel training orientation in the XXI century. Teaching physics in higher education. No. 14, 39 (1998).

3. R. Feynman, R. Leighton, M. Sands Feynman lectures on physics. 'Electricity and Magnetism, Moscow, Mir, v.5 (1987) $291 \mathrm{p}$.

4. G.Pein Physics of Oscillations and Waves. Moscow, Mir, (1979) 394 p.

5. Ju. De Broglie Introduction to Wave Mechanics. Kiev, State Scientific and Technical Institute of Ukraine (1934) 235 p.

6. E.Schrödinger Quantization as an eigenvalue problem. Variational Principles of Mechanics. Moscow, Gosizdat, Physics and Mathematics Literature (1959) 930 p.

7. J.K. Maxwell Articles and Speeches. Moscow, Nauka (1968) 423 p.

8. J.K. Maxwell Selected works on the theory of the electromagnetic field. Moscow, Gostekhizdat, (1954) 688 p..

9. G. Olson Dynamic analogies. Moscow, Gosizdat, in. Literature, (1997) 224 p.

10. E.Fermi Quantum Mechanics. Moscow, Mir (1988) $370 \mathrm{p}$.

11. S.Yu. Artsimovich, S.Lukyanov Movement of charged particles in electric and magnetic fields. Moscow, Nauka (1972) 228 p.

12. O.K. Kuvandikov, A. Zhumabaev The role and significance of analogy in the development and teaching of physics. Uzbek Physical Journal, 1998, No. 6, p. 7482. 